\title{
Variation in post-colonoscopy colorectal cancer across colonoscopy providers in English National Health Service: population based cohort study
}

\author{
Nicholas E Burr, ${ }^{1,2}$ Edmund Derbyshire, ${ }^{3}$ John Taylor, ${ }^{1}$ Simon Whalley, ${ }^{1}$ \\ Venkataraman Subramanian, ${ }^{4}$ Paul J Finan, ${ }^{1,5}$ Matthew D Rutter, ${ }^{6,7}$ Roland Valori, ${ }^{8}$ \\ Eva J A Morris ${ }^{1}$
}

For numbered affiliations see end of the article.

Correspondence to: N E Burr nick.burr@nhs.net (or@Nick_Burr1 on Twitter; ORCID 0000-0003-1988-2982) Additional material is published online only. To view please visit the journal online.

Cite this as: $B M J$ 2019;367:16090 http://dx.doi.org/10.1136/bmj.l6090

Accepted: 1 October 2019

\section{ABSTRACT \\ OBJECTIVES}

To quantify post-colonoscopy colorectal cancer (PCCRC) rates in England by using recent World Endoscopy Organisation guidelines, compare incidence among colonoscopy providers, and explore associated factors that could benefit from quality improvement initiatives.

DESIGN

Population based cohort study.

SETTING

National Health Service in England between 2005 and 2013.

POPULATION

All people undergoing colonoscopy and subsequently diagnosed as having colorectal cancer up to three years after their investigation (PCCRC-3yr).

MAIN OUTCOME MEASURES

National trends in incidence of PCCRC (within 6-36 months of colonoscopy), univariable and multivariable analyses to explore factors associated with occurrence, and funnel plots to measure variation among providers.

RESULTS

The overall unadjusted PCCRC-3yr rate was $7.4 \%$ (9317/126152), which decreased from $9.0 \%$ in

\section{WHAT IS ALREADY KNOWN ON THIS TOPIC}

Post-colonoscopy colorectal cancer (PCCRC) is a key indicator for the quality of a colonoscopy service, but information on its incidence has not been routinely available

Previous studies have used different approaches that make benchmarking and comparison of rates among providers unreliable

The World Endoscopy Organisation has recently produced standardised methods to calculate rates so that comparisons can be made among institutions and jurisdictions

\section{WHAT THIS STUDY ADDS}

This study used World Endoscopy Organisation methods and found wide variation in PCCRC rates within three years of investigation among colonoscopy providers in England

Rates declined from $9.0 \%$ for colonoscopies performed in 2005 to $6.5 \%$ for those performed in 2013 (P<0.01)

The English NHS bowel cancer screening programme rates were much lower $(3.6 \%)$ than those found for independent colonoscopy providers $(9.3 \%)$

By reducing the national PCCRC-3yr rate to an aspirational target of up to $3.6 \%$, many cancers could be prevented or diagnosed at an earlier stage
2005 to $6.5 \%$ in 2013 ( $\mathrm{P}<0.01)$. Rates were lower for colonoscopies performed under the NHS bowel cancer screening programme $(593 / 16640,3.6 \%)$, while they were higher for those conducted by non-NHS providers $(187 / 2009,9.3 \%)$. Rates were higher in women, in older age groups, and in people with inflammatory bowel disease or diverticular disease, in those with higher comorbidity scores, and in people with previous cancers. Substantial variation in rates among colonoscopy providers remained after adjustment for case mix.

\section{CONCLUSIONS}

Wide variation exists in PCCRC-3yr rates across NHS colonoscopy providers in England. The lowest incidence was seen in colonoscopies performed under the NHS bowel cancer screening programme. Quality improvement initiatives are needed to address this variation in rates and prevent colorectal cancer by enabling earlier diagnosis, removing premalignant polyps, and therefore improving outcomes.

\section{Introduction}

Colorectal cancer is a major public health problem in the United Kingdom. Each year, more than 40000 new patients are diagnosed as having the disease and there are around 16000 deaths. ${ }^{1}$ International comparisons have revealed that the survival rate of patients with colorectal cancer in the UK lags behind that attained by many of our economic neighbours. This finding is largely because of a high mortality rate soon after diagnosis, particularly among older people. The best survival rates are observed in patients who are diagnosed at an early stage. Therefore, outcomes can be improved by optimising the diagnostic process.

Colonoscopy is the main test for diagnosing colorectal cancer. This procedure also has the potential to prevent the disease by removing precancerous lesions, and so it is an important tool to help improve outcomes. $^{2}$ Unfortunately, the test is not $100 \%$ accurate and cancers can appear within months or years after a colonoscopy that is negative for cancer. The World Endoscopy Organisation defines these cases as post-colonoscopy colorectal cancers (PCCRCs). ${ }^{3}$ Evidence suggests that up to 700 patients are diagnosed as having PCCRCs each year in the English National Health Service (NHS). ${ }^{4}$ Therefore, reducing the incidence of these cancers is vital.

Colonoscopy services in the NHS are under pressure. Currently, over 650000 colonoscopies are undertaken each year, but demand is growing substantially and 
there is concern that the workforce needed to meet this demand is insufficient. Although major efforts have been made to ensure the NHS delivers high quality colonoscopy services, for example, the Joint Advisory Group on Gastrointestinal Endoscopy (JAG) accreditation scheme, not all providers have been mandated to participate. As a result, there is the potential for variation in the quality of colonoscopy services across the country; unless this variation is quantified, it cannot be addressed.

The British Society of Gastroenterology has proposed that PCCRC rates should be used as a benchmark for colonoscopy quality. ${ }^{5}$ Recently, the World Endoscopy Organisation ${ }^{6}$ provided methods to calculate rates so that comparisons can be made among institutions and jurisdictions. Our study aimed to apply these methods to compare PCCRC rates within three years of colonoscopy (PCCRC-3yr) among all providers, and to quantify variation in colonoscopy quality across the English NHS.

\section{Methods}

\section{Study population}

We obtained study data from the UK Colorectal Cancer Intelligence Hub's colorectal cancer data repository (CORECT-R), in which routine cancer datasets are linked to provide a rich resource of population based data. ${ }^{7}$ Our study population consisted of people who had undergone a colonoscopy in the English NHS between 1 January 2005 and 31 December 2013 and who subsequently developed colorectal cancer (International Classification of Diseases, 10th revision (ICD10) codes C18-20). We used linked inpatient and outpatient Hospital Episode Statistics (HES) data (OPCS4 codes H20-22), English NHS bowel cancer screening programme (BCSP), and National Cancer Registration and Analysis Service datasets available within the resource. The BCSP offers screening (through an initial guaiac based faecal occult blood test to determine eligibility for colonoscopy; soon to be replaced by a faecal immunochemical test) every two years to women and men aged 60-74. After age 74, people can request additional screening tests. ${ }^{8}$

The colonoscopy data included all procedures performed by NHS providers, private patients treated at NHS centres, and procedures performed by independent providers paid for by the NHS. We excluded tumours that involve the appendix (ICD10 C181) or those with a neuroendocrine, lymphoma, squamous, or melanoma morphology. Only adenocarcinoma subtypes were included because they are the main focus for detecting and preventing cancer after removal of serrated and adenomatous polyps. We limited our cohort to those with colorectal cancer that was diagnosed within three years of colonoscopy and with data extracted for analysis at colonoscopy level. The cohort was then split into three groups for analysis based on the year of colonoscopy: 2005-07, 2008-10, and 2011-13.

\section{PCCRC-3yr rate calculation}

Methods produced by the World Endoscopy Organisation were used to calculate the PCCRC-3yr rate. $^{6}$ We categorised colonoscopies into positive and negative tests according to whether a cancer was diagnosed within six months of the test. When a colorectal cancer occurred within the first six months after a colonoscopy, this was a true positive test; when a colorectal cancer appeared between six and 36 months after a colonoscopy, this was a false negative test. We defined cancers as detected cancers if they were preceded by a true positive colonoscopy and as a PCCRC-3yr if they were preceded by a false negative colonoscopy. This 6-36 month window for defining a PCCRC-3yr rate is advocated by the World Endoscopy Organisation so that institutions and jurisdictions can be compared by using the same metric. The definition does not require the index test to be negative for adenomas.

We appreciate that the terms false negative and true positive normally depend on a gold standard, which does not exist for the diagnosis of colorectal cancer and its precursors. Therefore, we assume that the gold standard is cancer diagnosed within three years of a colonoscopy. Some cancers that appear after a negative colonoscopy might be new, rapidly growing cancers or cancers arising from premalignant polyps. Thus, in this instance we use the term gold standard to refer to the potential of colonoscopy to detect or prevent cancers that will present within three years after the colonoscopy.

Some people in the cohort underwent multiple colonoscopies. For these people, we only included true positive and false negative colonoscopies that were recorded closest to the time of diagnosis, which is in accordance with World Endoscopy Organisation methods.

\section{Cohort characteristics}

We identified colonoscopies undertaken within the BCSP, but the indication for the procedure was otherwise unknown. However, other patient level data were available and so we investigated relevant factors that might increase the risk of PCCRC. These data included age at diagnosis and colonoscopy stratified into age bands ( $\leq 60,61-70,71-80$, and $>80$ ). We used these cut-off points because at the time of the study bowel cancer screening invitations began at age 60 . Other variables were sex, socioeconomic status (based on the income domain of the index of multiple deprivation 2007), ${ }^{9}$ and a comorbidity score based on diagnostic HES codes in the year before cancer diagnosis (subdivided into $0,1,2$, and $\geq 3$ ). The comorbidity score was the Charlson score, ${ }^{10}$ with the exception of malignancy because all patients had a diagnosis of colorectal cancer. We included several cancer related variables: stage, classified as stage I-IV, or unknown when data were missing; and colonic location of the tumour defined as caecum, right sided (ascending colon to transverse colon; C18.0-18.4), left sided (splenic flexure to rectosigmoid junction; C18.518.7 and C19.0), rectal (C20), or unspecified when no location was given (C18.8-18.9). We also recorded when cancers were diagnosed through an "emergency 
presentation" because this method of referral is associated with worse outcomes for colorectal cancer in the UK. ${ }^{11}$

Additionally, we identified people with a previous HES coded diagnosis of inflammatory bowel disease, Crohn's disease, ulcerative colitis (ICD10 codes K5051 ), or diverticular disease (ICD10 code K57). We also identified people with a previous diagnosis of colorectal cancer or previous colonoscopy, which was any colonoscopy performed before the test that diagnosed the colorectal cancer or the false negative test when the cancer was not diagnosed by colonoscopy.

\section{Statistical analyses}

We calculated the proportion of people with cancer who had true positive and false negative colonoscopies, and the PCCRC-3yr rate for each three year group according to World Endoscopy Organisation methods. We identified the healthcare provider that performed each colonoscopy by using the unique five digit codes recorded in HES data and from the screening centre in the BCSP. NHS organisations change over time (for example, hospital mergers), therefore historical organisations were mapped to current providers, as they existed on 1 January 2018. Some providers had low workloads, which made comparison of PCCRC3yr rates with other providers potentially unreliable. ${ }^{12}$ Therefore, we performed an a priori power calculation to determine the minimum number of cases that might be needed to detect an important difference among providers. We used a PCCRC-3yr rate of $7.5 \%$ for this calculation based on a previous rate of $8.6 \%$ from a study that covered an earlier time period ${ }^{4}$ and an assumption that this rate would have reduced. A doubling of this rate to $15 \%$ was deemed to be unacceptably high. Based on this number and 80\% power at the 0.05 significance level, a colonoscopy provider would need to detect 96 cancers in any given time period for there to be sufficient statistical power to be labelled as a significant outlier.

We calculated the PCCRC-3yr rate for each year in the study period, and used $\chi^{2}$ for trend as a significance test. Additional analyses were undertaken for subgroups: patients with inflammatory bowel disease; people who had colonoscopies as part of the BCSP; and a "non-surveillance" group that excluded people who had colonoscopies within the BCSP and those with a diagnosis of inflammatory bowel disease. We estimated the number of people with PCCRC if the unadjusted rate for each year in the study period was reduced to the 75 th centile as a potential benchmark for a minimum standard, and then at the rate achieved by the BCSP as an aspirational target. This would indicate the potential number of cancers that could be diagnosed earlier or prevented if the overall rate is improved to these levels.

We explored the change in PCCRC rate over time for each provider by grouping each unit in the earliest cohort (2005-07) into fifths based on the PCCRC-3yr rate. Similar fifths were then produced for the 2011-13 period and the change in fifths was compared for each colonoscopy provider.

We built multilevel, logistic regression models to determine factors associated with the occurrence of PCCRC-3yr. The models were structured to reflect that people could have undergone multiple colonoscopies, with the person fitted as a random effect. The dependent variable was the occurrence of PCCRC-3yr. Exploratory variables were year of colonoscopy, age group at colonoscopy, sex, index of multiple deprivation income category (fifths), comorbidity score, and a previous HES coded diagnosis of inflammatory bowel disease or diverticular disease, previous colorectal cancer, and previous colonoscopy; additionally, whether the colonoscopy was performed within the BCSP, independent sector, or a non-BCSP service.

We produced unadjusted and risk adjusted funnel plots by using these models and the Spiegelhalter method $^{13}$ to quantify variation in the rate of PCCRC$3 y r$ for each colonoscopy provider in each time period. Funnel plots are a useful graphical display for institutional variation; they are constructed as a scatterplot with superimposed control limits, which represent two and three standard deviations from the mean. The smaller the sample size, the wider the control limits. ${ }^{14}$ Providers who fall outside the control limits have PCCRC rates that are statistically significantly different from the national rate, independently of the case mix factors adjusted for and colonoscopy workload, and so indicate unwarranted variation. We also created unadjusted and risk adjusted histograms to show the PCCRC-3yr rate for each colonoscopy provider in each of the three time periods. Nonmodifiable risk factors were used for these models: year of colonoscopy, age group at colonoscopy, sex, index of multiple deprivation income category (fifths), comorbidity score, previous HES coded diagnosis of inflammatory bowel disease or diverticular disease, previous colorectal cancer, and previous colonoscopy; additionally whether the colonoscopy was undertaken within the BCSP or independent sector. All analyses were performed using Stata version 15 (StataCorp, College Station, TX).

\section{Patient and public involvement}

A patient and public group was involved at the design stage of the study. Results have been reported back to the group. The group will be involved in preparing dissemination materials which will be fed back to the colonoscopy providers and released through the national charities Bowel Cancer UK and Cancer Research UK.

\section{Results}

From an estimated population in England of over 55 million in 2016, ${ }^{15}$ our study included 126152 colonoscopies in 121402 people who were diagnosed as having colorectal cancer within three years of their investigation. In this population, 9317 (7.4\%) were classified as PCCRC-3yr. There was a statistically significant reduction in the PCCRC-3yr rate from 9.0\% 
for colonoscopies performed in 2005 to $6.5 \%$ for those performed in 2013 ( $\mathrm{P}<0.01)$ (fig 1). The PCCRC3 yr rate in people with inflammatory bowel disease was $38.3 \%$ for colonoscopies performed in 2005 and $35.5 \%$ for those performed in $2013(\mathrm{P}=0.24)$. For the BCSP colonoscopies, the rate increased from $2.7 \%$ in 2005 to $3.6 \%$ in 2013, but did not reach statistical significance $(\mathrm{P}=0.06)$. For colonoscopies performed in people with non-inflammatory bowel disease and those that were not conducted within the BCSP, there was a significant reduction in the rate from $8.2 \%$ in 2005 to $7.3 \%$ in $2013(\mathrm{P}<0.01)$. Most providers stayed within the same fifth of the PCCRC-3yr rate in the 2005-07 and 2011-13 groups (supplementary table S3).

Table 1 shows the proportion of detected cancers and the PCCRC-3yr rate in relation to the characteristics of the population and the colonoscopies they underwent. The PCCRC-3yr rate was higher after colonoscopies performed in the earlier study periods; in women; among people with a higher comorbidity score; in those with a previous HES code for inflammatory bowel disease or diverticular disease; in people with previous colorectal cancer; and with increasing numbers of previous colonoscopy tests. The PCCRC-3yr rate was higher in the oldest age group than in the youngest age group. Additionally, the rate was much lower in those who had colonoscopies as part of the BCSP (PCCRC$3 \mathrm{yr}$ rate $3.6 \%$ ) than in those who had colonoscopies performed by independent providers outside of the
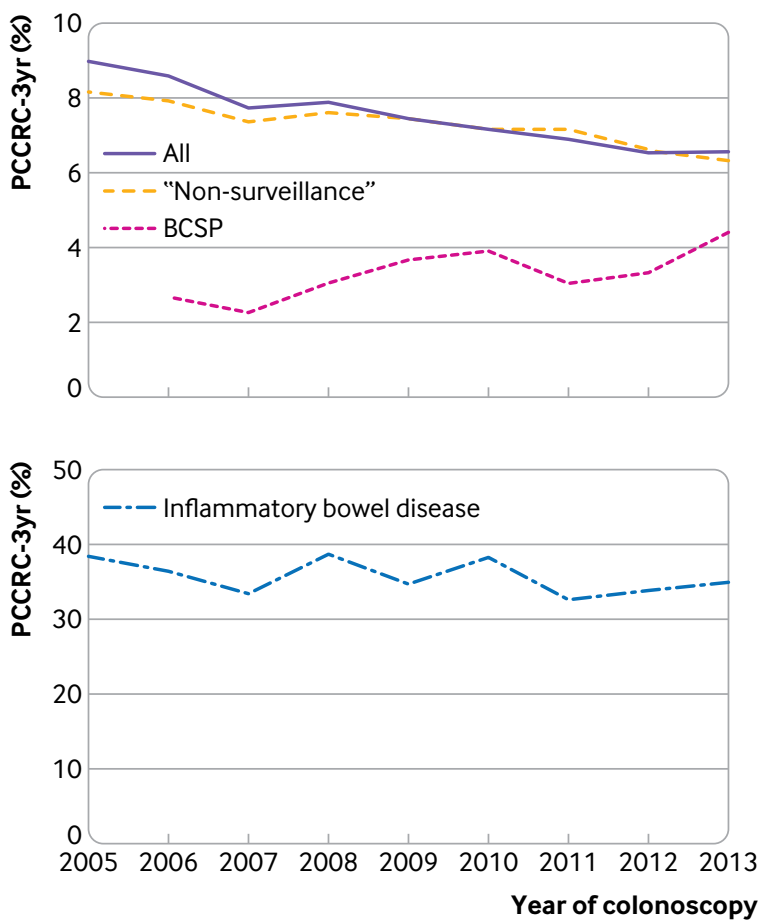

Fig 1 | Trends in unadjusted rates of post-colonoscopy colorectal cancer within three years of investigation (PCCRC-3yr) for each year of colonoscopy. Top panel: all colonoscopies, "non-surveillance," and UK bowel cancer screening programme (BCSP). Bottom panel: colonoscopies in people with an admission code for inflammatory bowel disease. "Non-surveillance" colonoscopies are those in people not diagnosed as having inflammatory bowel disease and those not performed within the BCSP
NHS (9.3\% across the entire study period). The PCCRC3yr rate was higher in people with early (stage I), late (stage IV), and unknown stage cancers, and in those with cancer in the proximal colon. Significantly more people were diagnosed as having PCCRC-3yr after an emergency presentation, which is associated with poorer outcomes.

After multivariable analysis for the factors which can be assessed at the time of colonoscopy, which excludes stage and colonic location, these associations were sustained and all remained statistically significant $(\mathrm{P}<0.01)$ (table 2).

\section{Institutional comparison}

A total of 135, 139, and 140 colonoscopy providers were operating in the English NHS during the time periods 2005-07, 2008-10, and 2011-13, respectively. Figure 2 and figure 3 show unadjusted and adjusted institutional comparisons for each of the time periods (see also table 3).

Across each time period we found significant variation in unadjusted and adjusted PCCRC-3yr rates. In 2011-13, unadjusted rates ranged from $3.2 \%$ to $16.4 \%$, and adjusted rates ranged from $3.2 \%$ to $12.8 \%$. Supplementary table 1 shows the centiles for the range of unadjusted PCCRC-3yr rates in 2011-13; the best performing 5\% had an unadjusted PCCRC-3yr rate of $4 \%$ and the worst performing $5 \%$ had a rate of $10.4 \%$.

In the unadjusted funnel plot for the years 2011-13, two providers were outside the upper $99.8 \%$ control limit, with 11 providers outside the upper $95 \%$ control limit, which indicates higher PCCRC-3yr rates than expected. In contrast, seven providers had rates below the lower 95\% control limit, which suggests they had lower PCCRC-3yr rates than expected. No providers were below the lower $99.8 \%$ control limit. After risk adjustment for the non-modifiable factors identified above, one provider was above the upper 99.8\% control limit, with 10 providers outside the upper $95 \%$ control limit. In contrast, there were five providers with rates below the lower $95 \%$ control limit. No providers had rates below the $99.8 \%$ control limit. Figure 2, figure 3, and table 3 show the results for the years 2005-07, 2008-10, and 2011-13.

We estimated the potential benefit of higher quality colonoscopy by calculating the reduction in PCCRC-3yr if a $6.7 \%$ rate, which was found in the 2011-13 cohort, was reduced across the study period. Supplementary figure S1 shows the results of these estimates. Overall, if we reduced the rate to $5.5 \%$ or $3.6 \%$ as achieved in the BCSP, for the entire study period there would potentially be 168 or 435 fewer patients with PCCRC each year, respectively.

\section{Discussion}

\section{Principal findings}

Our study uses World Endoscopy Organisation methods and robust population based data across the entire English NHS. Although PCCRC-3yr rates are declining, in the most recent cohort the rate was $6.5 \%$. We found a much lower PCCRC-3yr rate for 


\begin{tabular}{|c|c|c|c|c|}
\hline \multirow[t]{2}{*}{ Characteristic } & \multicolumn{3}{|c|}{$\begin{array}{l}\text { Colonoscopies with colorectal cancer diagnosed within } \\
\text { three years }\end{array}$} & \multirow[t]{2}{*}{ PCCRC-3yr rate (\%) } \\
\hline & Total & True positive & False negative & \\
\hline Total & 126152 & 116835 & 9317 & 7.4 \\
\hline \multicolumn{5}{|c|}{ Year of colonoscopy } \\
\hline 2005-07 & 31336 & $28711(25)$ & $2625(28)$ & 8.4 \\
\hline 2008-10 & 44165 & $40856(35)$ & $3309(36)$ & 7.5 \\
\hline 2011-13 & 50651 & $47268(40)$ & $3383(36)$ & 6.7 \\
\hline \multicolumn{5}{|c|}{ Age at colonoscopy } \\
\hline$\leq 60$ & 22185 & $20731(18)$ & $1454(16)$ & 6.6 \\
\hline $61-70$ & 38521 & $36153(31)$ & $2368(25)$ & 6.1 \\
\hline $71-80$ & 42833 & $39479(34)$ & $3354(36)$ & 7.8 \\
\hline 180 & 22613 & $20472(18)$ & $2141(23)$ & 9.5 \\
\hline \multicolumn{5}{|l|}{ Sex } \\
\hline Male & 73459 & $68344(58)$ & $5115(55)$ & 7.0 \\
\hline Female & 52693 & $48491(42)$ & $4202(45)$ & 8.0 \\
\hline \multicolumn{5}{|c|}{ IMD income category } \\
\hline Most affluent & 27180 & $25169(22)$ & $2011(22)$ & 7.4 \\
\hline 2 & 28331 & $26246(22)$ & $2085(22)$ & 7.4 \\
\hline 3 & 26479 & $24533(21)$ & $1946(21)$ & 7.3 \\
\hline 4 & 23857 & $22078(19)$ & 1779 (19) & 7.5 \\
\hline Least affluent & 20305 & $18809(16)$ & $1496(16)$ & 7.4 \\
\hline \multicolumn{5}{|c|}{ Charlson comorbidity score } \\
\hline 0 & 93684 & $87738(75)$ & $5946(64)$ & 6.3 \\
\hline 1 & 21576 & $19590(17)$ & $1986(21)$ & 9.2 \\
\hline 2 & 6438 & $5715(5)$ & $723(8)$ & 11.2 \\
\hline$\geq 3$ & 4454 & $3792(3)$ & $662(7)$ & 14.9 \\
\hline \multicolumn{5}{|c|}{ Inflammatory bowel disease } \\
\hline No & 123428 & $108893(98)$ & $8348(90)$ & 6.8 \\
\hline Yes & 2724 & $1756(2)$ & $968(10)$ & 35.5 \\
\hline \multicolumn{5}{|c|}{ Diverticular disease } \\
\hline No & 94437 & $88813(76)$ & $5624(60)$ & 6.0 \\
\hline Yes & 31715 & $28022(24)$ & $3693(40)$ & 11.6 \\
\hline \multicolumn{5}{|c|}{ Previous colorectal cancer } \\
\hline No & 124513 & $115708(99)$ & $8805(95)$ & 7.1 \\
\hline Yes & 1639 & $1127(1)$ & $512(5)$ & 31.2 \\
\hline \multicolumn{5}{|c|}{ Previous colonoscopy } \\
\hline No & 105972 & $100541(86)$ & $5431(58)$ & 5.1 \\
\hline Yes & 20180 & $16294(14)$ & $3886(42)$ & 19.3 \\
\hline \multicolumn{5}{|c|}{ Colonoscopy within BCSP } \\
\hline No & 109512 & $100788(86)$ & $8724(94)$ & 8.0 \\
\hline Yes & 16640 & $16047(14)$ & $593(6)$ & 3.6 \\
\hline \multicolumn{5}{|c|}{ Colonoscopy by independent provider } \\
\hline No & 124143 & $115013(98)$ & $9130(98)$ & 7.4 \\
\hline Yes & 2009 & $1822(2)$ & $187(2)$ & 9.3 \\
\hline \multicolumn{5}{|c|}{ Cancer characteristics } \\
\hline \multicolumn{5}{|l|}{ Stage: } \\
\hline 1 & 21474 & $19597(17)$ & $1877(20)$ & 8.7 \\
\hline II & 34605 & $32613(28)$ & $1992(21)$ & 5.8 \\
\hline III & 33240 & $31186(27)$ & $2054(22)$ & 6.2 \\
\hline IV & 13564 & $12252(10)$ & $1312(14)$ & 9.7 \\
\hline Unknown & 23269 & $21187(18)$ & $2082(22)$ & 8.9 \\
\hline \multicolumn{5}{|l|}{ Colonic location: } \\
\hline Rectum & 31492 & $29496(25)$ & $1996(21)$ & 6.3 \\
\hline Distal colon & 40783 & $38450(33)$ & $2333(25)$ & 5.7 \\
\hline Proximal colon & 27383 & $25143(22)$ & $2240(24)$ & 8.2 \\
\hline Caecum & 20978 & $19057(16)$ & $1921(21)$ & 9.2 \\
\hline Colon NOS & 5516 & $4689(4)$ & $827(9)$ & 15.0 \\
\hline
\end{tabular}

colonoscopies performed as part of the BCSP (3.6\%). If this aspirational rate had been achieved for the entire study period, more than 3900 patients with colorectal cancer could have been diagnosed earlier or received preventative treatment.
Although PCCRC-3yr rates fell during each successive time period, we still observed major discrepancies among providers, which persisted after adjustment for associated risk factors. This variation needs to be minimised to increase early diagnosis rates 
and improve colorectal cancer outcomes. PCCRC-3yr rates for colonoscopies undertaken by independent providers were higher than those reported for NHS providers. These independent providers are increasingly being used to meet the rising demand for colonoscopies.

We found a lower incidence of PCCRC-3yr for colonoscopies performed as part of the BCSP. All BCSP colonoscopies are performed within JAG accredited screening centres by colonoscopists who have undergone an additional accreditation process. ${ }^{16}$ BCSP colonoscopies are of a high standard, with high adenoma detection and caecal intubation rates. ${ }^{16}$ Screening colonoscopists adhere to strict performance criteria, including an unadjusted caecal intubation rate greater than $92 \%$ and an adenoma detection rate greater than $40 \%$. Adenoma detection rate correlates with PCCRC, therefore it is reasonable to assume that the high quality of colonoscopies in the BCSP contributes to the reduced PCCRC-3yr rates reported in this study. ${ }^{17}$ This important finding shows that when standards are applied rigorously, quality can improve. Another reason for the decrease in PCCRC-3yr could be because of improvements in endoscopic equipment, including adjuncts to improve detection. A recent randomised study showed increased adenoma number detection with newer generation endoscopes. ${ }^{18}$

In contrast to the BCSP, we found a higher PCCRC rate after colonoscopies undertaken in the independent sector. In recent years there has been a marked increase in demand for colonoscopies in the UK and NHS services have not been able to deliver the number of investigations required, principally because of workforce constraints. Therefore, the independent sector has been used to relieve the backlog of NHS waiting list procedures and we anticipate that they will have generally undertaken "low risk" colonoscopies for which the chance of finding cancer is reduced.

\begin{tabular}{|c|c|c|c|c|}
\hline \multirow[b]{2}{*}{ Characteristic } & \multicolumn{2}{|c|}{ Unadjusted } & \multicolumn{2}{|c|}{ Adjusted } \\
\hline & Odds ratio $(95 \% \mathrm{Cl})$ & $P$ value & Odds ratio $(95 \% \mathrm{Cl})$ & $P$ value \\
\hline Year of colonoscopy & & & 1 & \\
\hline 2005-07 & 1 & - & 1 & - \\
\hline $2008-10$ & 0.89 (0.84 to 0.93$)$ & $<0.01$ & $0.86(0.82$ to 0.91$)$ & $<0.01$ \\
\hline 2011-13 & 0.78 (0.74 to 0.83$)$ & $<0.01$ & $0.70(0.66$ to 0.74$)$ & $<0.01$ \\
\hline \multicolumn{5}{|l|}{ Age at colonoscopy } \\
\hline$\leq 60$ & 1 & - & 1 & - \\
\hline $61-70$ & 0.93 (0.87 to 1.00$)$ & 0.05 & $1.02(0.94$ to 1.09$)$ & 0.67 \\
\hline $71-80$ & 1.21 (1.14 to 1.29$)$ & $<0.01$ & $1.02(0.96$ to 1.10$)$ & 0.50 \\
\hline$>80$ & 1.49 (1.39 to 1.60$)$ & $<0.01$ & 1.17 (1.09 to 1.27$)$ & $<0.01$ \\
\hline \multicolumn{5}{|c|}{ 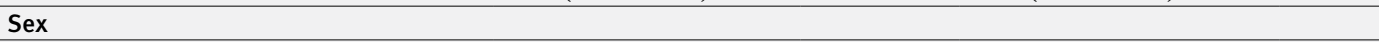 } \\
\hline Male & 1 & - & 1 & - \\
\hline Female & $1.16(1.11$ to 1.21$)$ & $<0.01$ & $1.15(1.10$ to 1.20$)$ & $<0.01$ \\
\hline \multicolumn{5}{|l|}{ IMD income category } \\
\hline Most affluent & 1 & - & 1 & - \\
\hline 2 & 0.99 (0.93 to 1.06$)$ & 0.86 & 0.98 (0.91 to 1.04$)$ & 0.50 \\
\hline 3 & 0.99 (0.93 to 1.06$)$ & 0.83 & $0.98(0.91$ to 1.05$)$ & 0.52 \\
\hline 4 & 1.01 (0.94 to (1.08) & 0.97 & 0.97 (0.91 to 1.04$)$ & 0.44 \\
\hline Least affluent & $1.00(0.93$ to 1.07$)$ & 0.9 & $0.93(0.86$ to 1.00$)$ & 0.27 \\
\hline \multicolumn{5}{|c|}{ Charlson comorbidity score } \\
\hline 0 & 1 & - & 1 & - \\
\hline 1 & 1.50 (1.42 to 1.58$)$ & $<0.01$ & $1.36(1.28$ to 1.44$)$ & $<0.01$ \\
\hline 2 & 1.87 (1.72 to 2.03$)$ & $<0.01$ & $1.62(1.48$ to 1.76$)$ & $<0.01$ \\
\hline$\geq 3$ & 2.58 (2.36 to 2.81$)$ & $<0.01$ & 2.17 (1.98 to 2.38$)$ & $<0.01$ \\
\hline \multicolumn{5}{|c|}{ Inflammatory bowel disease } \\
\hline No & 1 & - & 1 & - \\
\hline Yes & $7.60(7.00$ to 8.24$)$ & $<0.01$ & $4.93(4.50$ to 5.40$)$ & $<0.01$ \\
\hline \multicolumn{5}{|l|}{ Diverticular disease } \\
\hline No & 1 & - & 1 & - \\
\hline Yes & 2.08 (1.99 to 2.17$)$ & $<0.01$ & $1.88(1.79$ to 1.97$)$ & $<0.01$ \\
\hline \multicolumn{5}{|c|}{ Colonoscopy within BCSP } \\
\hline No & 1 & - & 1 & - \\
\hline Yes & $0.4(0.39$ to 0.46$)$ & $<0.01$ & $0.68(0.62$ to 0.74$)$ & $<0.01$ \\
\hline \multicolumn{5}{|c|}{ Colonoscopy by independent provider } \\
\hline No & 1 & - & 1 & - \\
\hline Yes & $1.29(1.11$ to 1.51$)$ & $<0.01$ & $1.63(1.39$ to 1.91$)$ & $<0.01$ \\
\hline \multicolumn{5}{|c|}{ Previous colorectal cancer } \\
\hline No & 1 & - & 1 & - \\
\hline Yes & $5.95(5.35$ to 6.62$)$ & $<0.01$ & 2.24 (2.00 to 2.52$)$ & $<0.01$ \\
\hline \multicolumn{5}{|l|}{ Previous colonoscopy } \\
\hline No & 1 & - & 1 & - \\
\hline Yes & $4.41(4.22$ to 4.62$)$ & $<0.01$ & $3.29(3.13$ to 3.46$)$ & $<0.01$ \\
\hline
\end{tabular}


We suggest that people at higher risk of developing PCCRC (for example, those with inflammatory bowel disease, genetic conditions predisposing them to colorectal cancer, or large polyps) will have remained under NHS care and so the higher rates of PCCRC in the independent sector might be a cause for concern; however, more detailed data are required to fully quantify the risks. Additional research into provider characteristics that influence colonoscopy outcomes would provide valuable information on how to optimise NHS colonoscopy services. For example, do outcomes differ among providers who do and do not deliver $\mathrm{JAG}^{19}$ authorised colonoscopy training? While we were not able to perform these analyses in our study, further studies using new data sources, such as the new National Endoscopy Database and the Private
Healthcare Information Network, are proposed to deliver this additional information.

We found that it is important to determine risk factors and define high risk groups so that endoscopists are more aware of the potential for PCCRC. Our data support established risk factors, including increasing age at colonoscopy, ${ }^{20}$ female sex, ${ }^{20}{ }^{21}$ more comorbidities, ${ }^{22}$ diverticular disease, ${ }^{22} 23$ and inflammatory bowel disease. $^{24}$ Previous colorectal cancer and multiple previous colonoscopies also had strong associations with PCCRC rates. Some of these factors might be associated with incomplete colonoscopy, which is a strong predictor of PCCRC risk. ${ }^{20}$ In this event, it is important that a timely repeat procedure or alternative test is performed, such as cross sectional imaging if clinically appropriate.
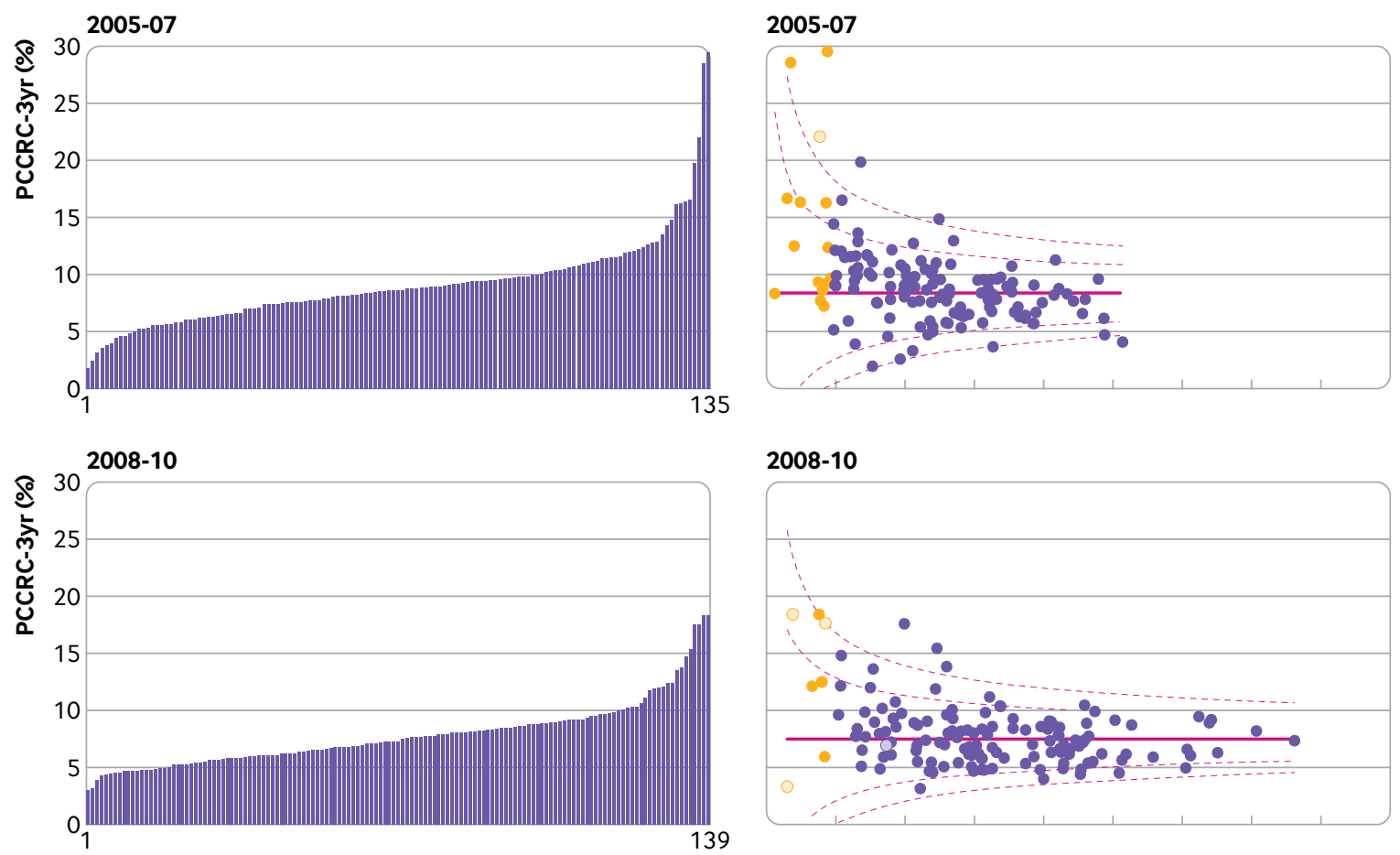

2008-10

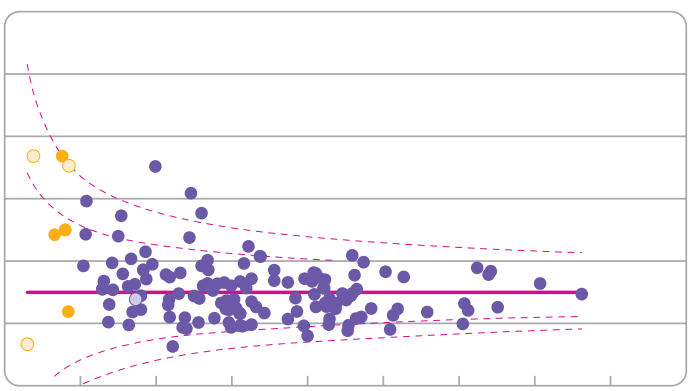

2011-13

2011-13
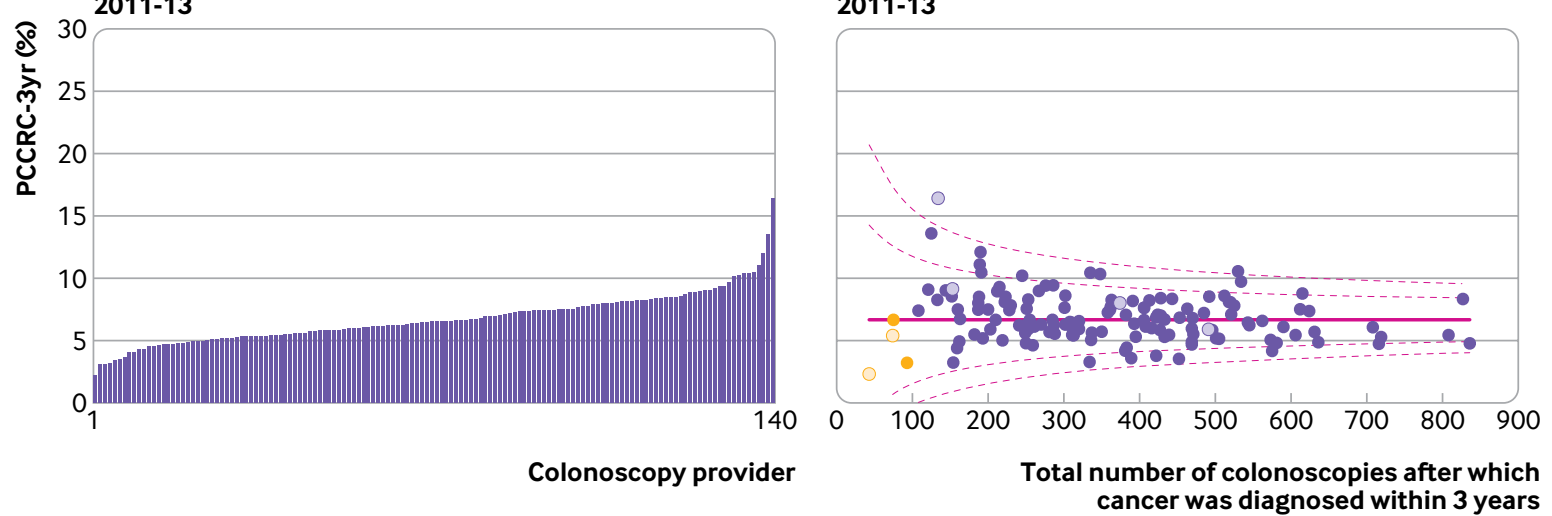

Fig 2 | Unadjusted variation in rates of post-colonoscopy colorectal cancer within three years of investigation (PCCRC-3yr) by provider for 2005-07, 2008-10, and 2011-13. In funnel plots each dot represents an individual colonoscopy provider. Dashed lines represent $95 \%$ and $99.8 \%$ control limits outside national PCCRC-3yr rate (solid line). X axis is number of detected cancers plus PCCRC-3yr cancers diagnosed in the period. Yellow dots indicate providers who diagnosed less than 96 cancers in the given period. Hollow dots represent independent colonoscopy providers 

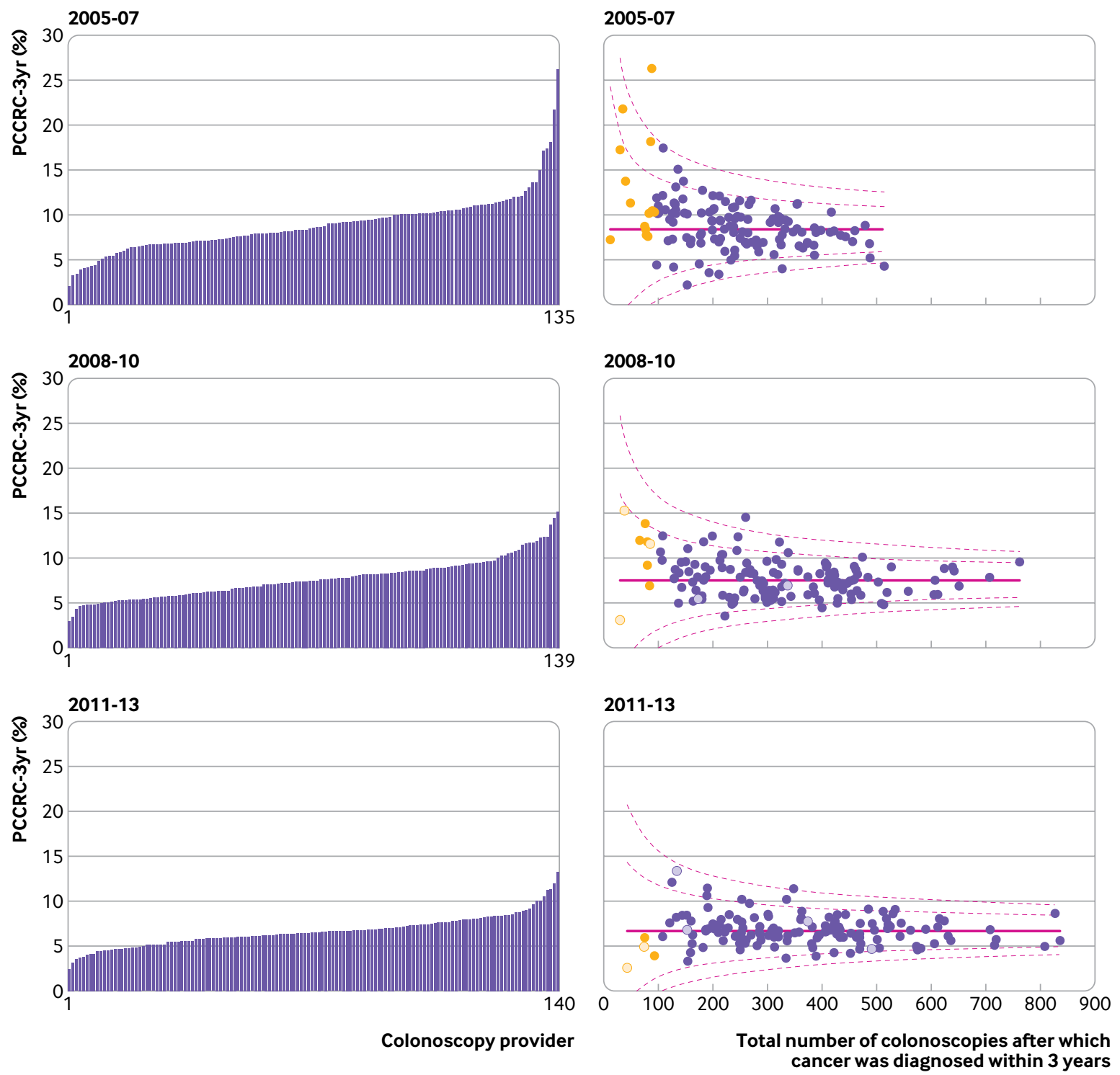

Fig 3 | Adjusted variation in rates of post-colonoscopy colorectal cancer within three years of investigation (PCCRC-3yr) by provider for 2005-07, 2008-10, and 2011-13. In funnel plots each dot represents an individual colonoscopy provider. Dashed lines represent $95 \%$ and $99.8 \%$ control limits outside national PCCRC-3yr rate (solid line). $X$ axis is number of detected cancers plus PCCRC-3yr cancers diagnosed in the period. Adjusted for non-modifiable risk factors: year of colonoscopy, age group at colonoscopy, sex, index of multiple deprivation income category (fifths), comorbidity score, previous Hospital Episode Statistics coded diagnosis of inflammatory bowel disease or diverticular disease, previous colorectal cancer, previous colonoscopy, and whether the colonoscopy was within English NHS bowel cancer screening programme or by independent provider. Yellow dots indicate providers who diagnosed less than 96 cancers in the given period. Hollow dots represent independent colonoscopy providers

The PCCRC-3yr rate for people with inflammatory bowel disease was over three times that of the entire study cohort, an association that has been reported in previous studies. ${ }^{24} 25$ Despite a significant improvement in rates in people without inflammatory bowel disease, the PCCRC-3yr rate in people with inflammatory bowel disease remained constant throughout our study period $(\mathrm{P}=0.24)$. Societal guidelines now advocate surveillance on an annual basis for some people with inflammatory bowel disease who are at high risk. While these UK guidelines were published in $2010,{ }^{26}$ they do not seem to have impacted on the PCCRC-3yr rate (although the data are relatively immature, with colonoscopy data from only three years after their introduction). Additional work to monitor guideline implementation and impact on PCCRC rates is warranted, not least because colorectal cancer is a serious complication of inflammatory bowel disease, and surveillance colonoscopy is a considerable burden for patients and healthcare providers. In the context of surveillance, detection of some patients with PCCRC could reflect the success of the procedure in identifying colorectal cancer early. Therefore, with more colonoscopies being performed, an increase in PCCRC rates will be inevitable, although it is unlikely that this accounts entirely for the increased PCCRC rates found in our study. Despite guidelines for surveillance, ${ }^{26}$ 
unfortunately these are poorly adhered to. ${ }^{27}$ Quality measures will need to be addressed, which should be the focus of future studies.

\section{Strengths and limitations}

Previous studies have shown the rates of PCCRC are highly sensitive to the method used for calculation and the quality of the data to which that method is applied. ${ }^{4}$ It is vital to have consistent methods to enable reliable benchmarking and comparison of rates. Such an approach would identify what might be possible, but also would highlight areas of underperformance that could benefit from quality improvement interventions. Our study used standardised methods from the World Endoscopy Organisation to calculate PCCRC-3yr rates from a population based dataset that includes all cancers diagnosed in England and all colonoscopies associated with cancer undertaken as part of the screening programme or to investigate symptoms. It is not feasible to perform these analyses on single centre or local datasets because there is often a time lag of several years between the negative test and subsequent pathology. Furthermore, people might be diagnosed as having cancer in a different centre to where the colonoscopy was performed, and therefore, not be captured using local data. National datasets circumvent this problem to a large extent.

The study is potentially limited because the data are from routinely collected clinical codes, and therefore are subject to ascertainment bias. We believe this risk to be low because previous validation studies have shown 96\% accuracy for routine data collected in HES and over 99\% accuracy in the National Cancer Registry and Analysis Service. ${ }^{28} 29$ The dataset is taken from an NHS administrative dataset that is used for reimbursement and so we expect the numbers of procedures not included in this dataset to be extremely low; however, this possibility cannot be discounted entirely. In addition, there will be a small number

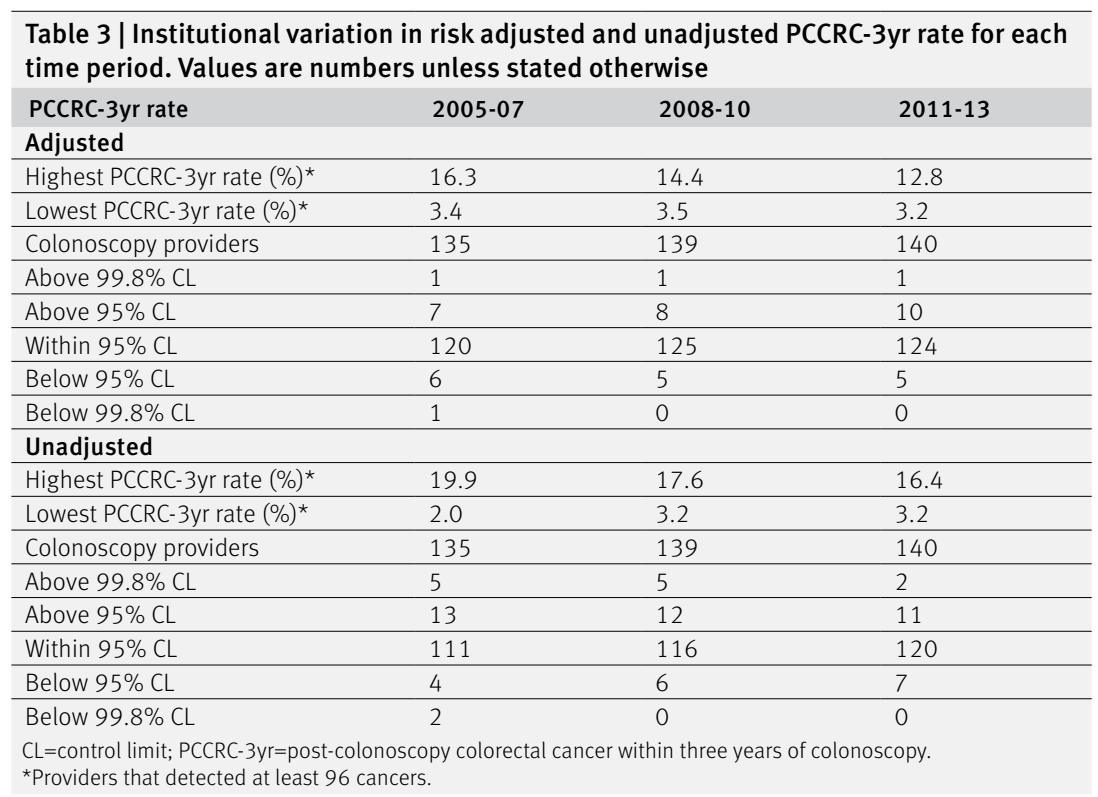

of colonoscopies undertaken outside of the NHS by independent providers.

Because we based the study on administrative data, there were important potential causal factors that we could not consider in these analyses. For example, details on whether lesions were incompletely resected, ${ }^{30}$ the presence of large or multiple adenomas, the extent of bowel preparation, failure to book or non-compliance with recommendations for repeat shorter interval testing are all relevant. Unfortunately, such data are not routinely collected in English population datasets. Service level factors exist at each institution that could account for some of the variation seen. Additionally, some institutions perform more procedures with a higher risk of colorectal cancer and therefore PCCRC, such as inflammatory bowel disease surveillance and surveillance after the endoscopic removal of large polyps or early cancers. These variables should be considered in any future research into the cause of PCCRC.

\section{Conclusions and policy implications}

Overall, there has been a sustained improvement in the PCCRC-3yr rate in England. This probably reflects the national initiatives put in place to improve the quality of colonoscopy in England since 2003, overseen initially by the National Endoscopy Team, ${ }^{31}$ and subsequently by the JAG and the British Society of Gastroenterology. For example, most endoscopy services in England are now participating in JAG accreditation, which involves achieving certain standards for service and training, including monitoring performance of colonoscopy and implementing interventions to address poor performance. Additionally, there has been a national colonoscopy training programme since $2003 .{ }^{31}$ There is some evidence that these interventions have improved colonoscopy performance, ${ }^{32-34}$ particularly completion of the procedure and appropriate adenoma detection rates, but further work is required to confirm their impact. It is also noteworthy that providers outside the NHS, which have only recently engaged in JAG accreditation, ${ }^{35}$ had a higher overall rate of PCCRC-3yr than NHS providers. It will be important to determine if gaining JAG accreditation helps to close the gap in PCCRC rates between NHS and non-NHS providers. A recent study from Canada, where no such quality improvement initiatives have been mandated, found no reduction in the PCCRC-3yr rate in those aged 50-74 between 1996 and 2010. ${ }^{36}$

Benchmarks need to be set for minimum acceptable standards and aspirational targets. These benchmarks have not been defined for PCCRC-3yr rates. The $25 \%$ centile from the range of unadjusted PCCRC-3yr rates was $5.5 \%$ and the BCSP rate was $3.6 \%$; these levels would be reasonable benchmarks for a minimum standard and aspirational target, respectively. Reduced rates could be achievable because colorectal cancer detected under the BCSP are smaller lesions, and therefore more likely to be missed. ${ }^{37}$ Furthermore, rates were falling up to the 
end of 2013; if this trend has continued, recent rates are already likely to be lower.

We need to define causal factors for PCCRC to target people most at risk and implement quality improvement interventions. A large study of PCCRC risk factors found that in a cohort of over 1000 patients with PCCRC, $46 \%$ had at least one factor that might have been avoidable. ${ }^{20}$ In the UK, a recent cohort study by Anderson and colleagues found that approximately $70 \%$ of PCCRCs might be because of avoidable factors, such as missed or incompletely resected lesions at previous colonoscopy. ${ }^{38}$ These results suggest that despite the improvements over time observed in our study, further reduction in PCCRC-3yr rates is possible.

Serious potential implications exist after a failed test, not limited to colorectal cancer investigations. After a false negative test patients and healthcare professionals might ignore significant symptoms, and delay or avoid repeat investigation. False negative tests also represent a missed opportunity to prevent a future cancer, and the patient might have been spared the consequences of the diagnosis. Potential costs are incurred for patients and healthcare funders and providers of a false negative test: a second colonoscopy or other test might be required; treatment might have to be given for cancer that could have been unnecessary; and cancers are probably more advanced and might have metastasised. Finally, there is the psychological impact of a delayed diagnosis; even if the prognosis is unaffected, patients and their relatives will probably believe their outlook is worse because of the delay.

There is robust evidence that endoscopic screening prevents colorectal cancer, ${ }^{39}$ and the removal of adenomatous polyps reduces death from colorectal cancer. $^{40}$ However, by improving the quality of colonoscopy there is the potential for over diagnosis $^{41}$ because polyps will be detected that prompt surveillance in some people who will not die from colorectal cancer. Reassuringly, recent studies have shown a low risk of colorectal cancer in those with small polyps found at their index test. ${ }^{42}{ }^{43}$ As a result of this evidence, surveillance guidelines might change to try and reduce the number of unnecessary colonoscopies.

In conclusion, we suggest that the PCCRC-3yr rate is a key performance indicator of the quality of colonoscopy. A minimum standard of up to $5.5 \%$ and an aspirational target of up to $3.6 \%$ could be applied as quality standards. Substantial unwarranted variation in PCCRC-3yr rates exists among colonoscopy providers in England. PCCRC is largely avoidable and targeted measures are required to reduce rates for all colonoscopy providers, improve earlier detection, and reduce mortality rates from this preventable disease.

\section{AUTHOR AFFILIATIONS}

${ }^{1}$ Cancer Epidemiology Group, Institute of Cancer and Pathology and Institute of Data Analytics, University of Leeds, Leeds LS2 9JT, UK ${ }^{2}$ Mid Yorkshire Hospitals NHS Trust, Pinderfields General Hospital, Wakefield, UK

${ }^{3}$ Royal Liverpool and Broadgreen University Hospitals NHS Trust, Liverpool, UK
${ }^{4}$ Leeds Teaching Hospitals NHS Trust, St James's University Hospital, Leeds, UK

${ }^{5}$ John Goligher Colorectal Unit, St James's University Hospital, Leeds, UK ${ }^{6}$ University Hospital of North Tees, Hardwick, Stockton on Tees, UK ${ }^{7}$ Northern Institute for Cancer Research, Newcastle University, Newcastle, UK

${ }^{8}$ Gloucestershire Hospitals NHS Foundation Trust, Gloucester, UK This project involves data derived from patient level information collected by the NHS, as part of the care and support of patients with cancer. The data are collated, maintained, and quality assured by the National Cancer Registration and Analysis Service, which is part of Public Health England (PHE). Access to the data was facilitated by the PHE Office for Data Release.

Contributors: NEB and ED are joint first authors. MDR, RV, and EIAM contributed equally and are joint senior authors. EJAM, RV, MDR, NEB, and ED conceived and designed the study. SW, NEB, and EM carried out the data linkage and preparation of the extracts of data from the CORECT-R within the National Colorectal Cancer Intelligence Hub. NEB $\mathrm{JT}$, and EM undertook the statistical analysis. All authors assisted in the interpretation of the data, drafting the manuscript, and approved the final version. NEB, EM, RV, and MDR are the guarantors. The corresponding author attests that all listed authors meet authorship criteria and that no others meeting the criteria have been omitted. Funding: This work is supported by the Bobby Moore Fund, Cancer Research UK, Yorkshire Cancer Research, and Crohn's and Colitis UK (C23434/A23706/M16-4). The Bobby Moore Fund, Cancer Research UK, Yorkshire Cancer Research and Crohn's and Colitis UK funded the work, but they were not involved in the collection, analysis, or interpretation of data, or in the writing or submitting of this report. The corresponding author had full access to all data and final responsibility for the decision to submit for publication.

Competing interests: All authors have completed the ICMJE uniform disclosure form at www.icmje.org/coi disclosure.pdf and declare: support from the Bobby Moore Fund, Cancer Research UK, Yorkshire Cancer Research, and Crohn's and Colitis UK for the submitted work; no financial relationships with any organisations that might have an interest in the submitted work in the previous three years, no other relationships or activities that could appear to have influenced the submitted work.

Ethical approval: The CORECT-R resource, and analyses based upon the data within it, has received approval from the South West-Central Bristol research ethics committee (18/SW/0134).

Data sharing: This project involves data derived from patient level information collected by the NHS, as part of the care and support of patients with cancer.

The lead author (NEB) affirms that the manuscript is an honest, accurate, and transparent account of the study being reported; that no important aspects of the study have been omitted; and that any discrepancies from the study as planned (and, if relevant, registered) have been explained.

This is an Open Access article distributed in accordance with the terms of the Creative Commons Attribution (CC BY 4.0) license, which permits others to distribute, remix, adapt and build upon this work, for commercial use, provided the original work is properly cited. See: http://creativecommons.org/licenses/by/4.0/.

1 Cancer Research UK. Bowel Cancer Statistics 2016. Accessed 01/02/2019. https://www.cancerresearchuk.org/healthprofessional/cancer-statistics/statistics-by-cancer-type/bowelcancer.

2 Winawer SJ, Zauber AG, Ho MN, et al, The National Polyp Study Workgroup. Prevention of colorectal cancer by colonoscopic polypectomy. N Engl I Med 1993;329:1977-81. doi:10.1056/ NEJM199312303292701

3 Rabeneck L, Paszat LF. Circumstances in which colonoscopy misses cancer. Frontline Gastroenterol 2010;1:52-8. doi:10.1136/ fg.2009.000257

4 Morris EJA, Rutter MD, Finan PJ, Thomas JD, Valori R. Post-colonoscopy colorectal cancer (PCCRC) rates vary considerably depending on the method used to calculate them: a retrospective observational population-based study of PCCRC in the English National Health Service. Gut 2015;64:1248-56. doi:10.1136/gutjnl-2014-308362

5 Rees C), Thomas Gibson S, Rutter MD, et al, British Society of Gastroenterology, the Joint Advisory Group on GI Endoscopy, the Association of Coloproctology of Great Britain and Ireland. UK key performance indicators and quality assurance standards for colonoscopy. Gut 2016;65:1923-9. doi:10.1136/ gutjnl-2016-312044 
6 Rutter MD, Beintaris I, Valori R, et al. World Endoscopy Organization consensus statements on post-colonoscopy and post-imaging colorectal cancer. Gastroenterology 2018;155:909-925.e3. doi:10.1053/j.gastro.2018.05.038

7 UK Bowel Cancer Intelligence Hub. University of Leeds; 2018. https:// bci.leeds.ac.uk/.

8 Logan RFA, Patnick J, Nickerson C, Coleman L, Rutter MD, von Wagner C, English Bowel Cancer Screening Evaluation Committee. Outcomes of the Bowel cancer screening programme (BCSP) in England after the first 1 million tests. Gut 2012;61:1439-46. doi:10.1136/ gutjnl-2011-300843

9 Noble M, McLennan D, Wilkinson K. The English Indices of Deprivation 2007. March 2008. Communities and Local Government: London. http://geoconvert.mimas.ac.uk/help/imd-2007-manual.pdf

10 Charlson ME, Pompei P, Ales KL, MacKenzie CR. A new method of classifying prognostic comorbidity in longitudinal studies: development and validation. J Chronic Dis 1987;40:373-83. doi:10.1016/0021-9681(87)90171-8

11 Elliss-Brookes L, McPhail S, Ives A, et al. Routes to diagnosis for cancer - determining the patient journey using multiple routine data sets. Br J Cancer 2012;107:1220-6. doi:10.1038/bjc.2012.408

12 Walker K, Neuburger J, Groene O, Cromwell DA, van der Meulen J. Public reporting of surgeon outcomes: low numbers of procedures lead to false complacency. Lancet 2013;382:1674-7. doi:10.1016/ S0140-6736(13)61491-9

13 Spiegelhalter DJ. Funnel plots for comparing institutional performance. Stat Med 2005;24:1185-202. doi:10.1002/sim.1970

14 Mayer EK, Bottle A, Rao C, Darzi AW, Athanasiou T. Funnel plots and their emerging application in surgery. Ann Surg 2009;249:376-83. doi:10.1097/SLA.0b013e31819a47b1

15 Estimates of the population for the UK, England and Wales, Scotland and Northern Ireland, UK Government, Office for National Statistics. 2016. https://www.ons.gov.uk/

16 Lee TJ, Rutter MD, Blanks RG, et al. Colonoscopy quality measures: experience from the NHS bowel cancer screening programme. Gut 2012;61:1050-7. doi:10.1136/gutjnl-2011-300651

17 Kaminski MF, Regula J, Kraszewska E, et al. Quality indicators for colonoscopy and the risk of interval cancer. N Engl J Med 2010;362:1795-803. doi:10.1056/NEJMoa0907667

18 Zimmermann-Fraedrich K, Groth S, Sehner S, et al. Effects of two instrument-generation changes on adenoma detection rate during screening colonoscopy: results from a prospective randomized comparative study. Endoscopy 2018;50:878-85. doi:10.1055/a-0607-2636

19 Siau K, Green JT, Hawkes ND, et al. Impact of the Joint Advisory Group on Gastrointestinal Endoscopy (JAG) on endoscopy services in the UK and beyond. Frontline Gastroenterol 2019;10:93-106. doi:10.1136/ flgastro-2018-100969

20 Tollivoro TA, Jensen CD, Marks AR, et al. Index colonoscopy-related risk factors for postcolonoscopy colorectal cancers. Gastrointest Endosc 2019;89:168-176.e3, e3. doi:10.1016/j.gie.2018.08.023

21 Brenner H, Chang-Claude J, Seiler CM, Hoffmeister M. Interval cancers after negative colonoscopy: population-based case-control study. Gut 2012;61:1576-82. doi:10.1136/gutjnl-2011-301531

22 Erichsen R, Baron JA, Stoffel EM, Laurberg S, Sandler RS, Sørensen HT. Characteristics and survival of interval and sporadic colorectal cancer patients: a nationwide population-based cohort study. Am J Gastroenterol 2013;108:1332-40. doi:10.1038/ajg.2013.175

23 Cooper GS, Xu F, Barnholtz Sloan JS, Schluchter MD, Koroukian SM Prevalence and predictors of interval colorectal cancers in medicare beneficiaries. Cancer 2012;118:3044-52. doi:10.1002/cncr.26602

24 Mooiweer E, van der Meulen-de Jong AE, Ponsioen CY, et al, Dutch Initiative on Crohn's and Colitis. Incidence of interval colorectal cancer among inflammatory bowel disease patients undergoing regular colonoscopic surveillance. Clin Gastroenterol Hepatol 2015;13:1656-61. doi:10.1016/j.cgh.2015.04.183

25 Rutter MD, Saunders BP, Wilkinson KH, et al. Thirty-year analysis of a colonoscopic surveillance program for neoplasia in ulcerative colitis. Gastroenterology 2006;130:1030-8. doi:10.1053/j. gastro.2005.12.035
26 Cairns SR, Scholefield JH, Steele RJ, et al, British Society of Gastroenterology, Association of Coloproctology for Great Britain and Ireland. Guidelines for colorectal cancer screening and surveillance in moderate and high risk groups (update from 2002). Gut 2010;59:666-89. doi:10.1136/gut.2009.179804

27 GasTRIN-NoW. P237 Regional IBD surveillance endoscopy north west (RISE NoW): an audit of surveillance colonoscopy practice in inflammatory bowel disease in north-west England. Journal of Crohn's and Colitis 2019;13(supplement_1):S215-16. doi:10.1093/ ecco-jcc/jjy222.361

28 Burns EM, Rigby E, Mamidanna R, et al. Systematic review of discharge coding accuracy. J Public Health (Oxf) 2012;34:138-48. doi:10.1093/pubmed/fdr054

29 Møller H, Richards S, Hanchett N, et al. Completeness of case ascertainment and survival time error in English cancer registries: impact on 1-year survival estimates. Br J Cancer 2011;105:170-6. doi:10.1038/bjc.2011.168

30 Forsberg A, Hammar U, Ekbom A, Hultcrantz R. Post-colonoscopy colorectal cancers in Sweden: room for quality improvement. Eur J Gastroenterol Hepatol 2017;29:855-60. doi:10.1097/ MEG.0000000000000884

31 Valori R. Quality improvements in endoscopy in England. Tech Gastrointest Endosc 2012;14:63-72. doi:10.1016/j. tgie.2011.11.001

32 Gavin DR, Valori RM, Anderson JT, Donnelly MT, Williams JG, Swarbrick ET. The national colonoscopy audit: a nationwide assessment of the quality and safety of colonoscopy in the UK. Gut 2013;62:242-9. doi:10.1136/gutjnl-2011-301848

33 Valori RM, Damery S, Gavin DR, et al. A new composite measure of colonoscopy: the Performance Indicator of Colonic Intubation (PICl). Endoscopy 2018;50:40-51. doi:10.1055/s-0043-115897

34 Valori RM, Thomas-Gibson S. Commentary: Accrediting colonoscopy services and colonoscopists for screening makes a difference. Colorectal Dis 2018;20:0283-5. doi:10.1111/codi.14374

35 Joint Advisory Group on Gl endoscopy. Participating services. Accessed 01/02/2019. https://www.thejag.org.uk/RegisteredUnits.aspx.

36 Murthy SK, Benchimol El, Tinmouth J, et al. Temporal trends in postcolonoscopy colorectal cancer rates in 50- to 74-yearold persons: a population-based study. Gastrointest Endosc 2018;87:1324-1334.e4, e4. doi:10.1016/j.gie.2017.12.027

37 Plumb AA, Halligan S, Nickerson C, et al. Use of CT colonography in the English bowel cancer screening programme. Gut 2014;63:96473. doi:10.1136/gutjnl-2013-304697

38 Anderson R, Valori R. PTH-009 Avoidable factors are identified in $70 \%$ of post colonoscopy colorectal cancers (PCCRCS). Gut 2018:67:A16-7. doi:10.1136/gutinl-2018-BSGAbstracts.31

39 Atkin WS, Edwards R, Kralj-Hans I, et al, UK Flexible Sigmoidoscopy Trial Investigators. Once-only flexible sigmoidoscopy screening in prevention of colorectal cancer: a multicentre randomised controlled trial. Lancet 2010;375:1624-33. doi:10.1016/S01406736(10)60551-X

40 Løberg M, Kalager M, Holme $\emptyset$, Hoff G, Adami HO, Bretthauer M. Long-term colorectal-cancer mortality after adenoma removal. N Engl J Med 2014;371:799-807. doi:10.1056/NEJMoa1315870

41 Brodersen J, Schwartz LM, Heneghan C, O’Sullivan JW, Aronson JK, Woloshin S. Overdiagnosis: what it is and what it isn't. BMJ Evid Based Med 2018;23:1-3. doi:10.1136/ebmed-2017-110886

42 Lieberman D, Sullivan BA, Hauser ER, et al. Baseline colonoscopy findings associated with 10-year outcomes in a screening cohort undergoing colonoscopy surveillance. Gastroenterology 2019;S0016-5085(19)41149-9. doi:10.1053/j. gastro.2019.07.052

43 He X, Hang D, Wu K, et al. Long-term risk of colorectal cancer after removal of conventional adenomas and serrated polyps. Gastroenterology 2019;156:S0016-5085(19)41086-X. doi:10.1016/S0016-5085(19)37161-6

Web appendix: Supplementary materials Web appendix: Visual summary 